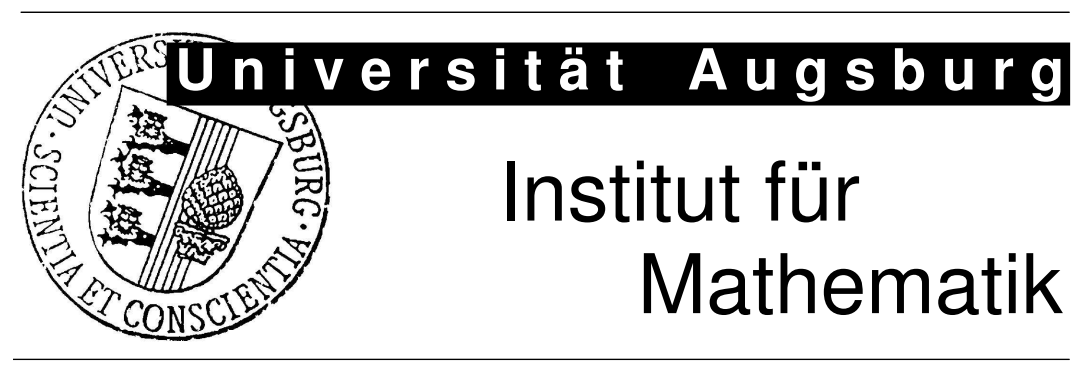

Stéphane Bordas, Ronald H.W. Hoppe, Svetozara I. Petrova Mechanical Failure in Microstructural Heterogeneous Materials 


\section{Impressum:}

Herausgeber:

Institut für Mathematik

Universität Augsburg

86135 Augsburg

http://www . math. uni-augsburg.de/forschung/preprint/

ViSdP:

Ronald H.W. Hoppe

Institut für Mathematik

Universität Augsburg

86135 Augsburg

Preprint: Sämtliche Rechte verbleiben den Autoren (C) 2007 


\title{
Mechanical Failure in Microstructural Heterogeneous Materials*
}

\author{
Stéphane Bordas ${ }^{1}$, Ronald H.W. Hoppe ${ }^{2,3}$, and Svetozara I. Petrova ${ }^{2,4}$ \\ ${ }^{1}$ Laboratory of structural and continuum mechanics, EPFL, Lausanne, Switzerland \\ ${ }^{2}$ Institute of Mathematics, University of Augsburg, 86159 Augsburg, Germany \\ ${ }^{3}$ Department of Mathematics, University of Houston, TX 77204-3008, USA \\ ${ }^{4}$ Institute for Parallel Processing, BAS, Block 25A, 1113 Sofia, Bulgaria
}

\begin{abstract}
Various heterogeneous materials with multiple scales and multiple phases in the microstructure have been produced in the recent years. We consider a mechanical failure due to the initiation and propagation of cracks in places of high pore density in the microstructures. A multi-scale method based on the asymptotic homogenization theory together with the mesh superposition method ( $s$-version of FEM) is presented for modeling of cracks. The homogenization approach is used on the global domain excluding the vicinity of the crack where the periodicity of the microstructures is lost and this approach fails. The multiple scale method relies on efficient combination of both macroscopic and microscopic models. The mesh superposition method uses two independent (global and local) finite element meshes and the concept of superposing the local mesh onto the global continuous mesh in such a way that both meshes not necessarily coincide. The homogenized material model is considered on the global mesh while the crack is analyzed in the local domain (patch) which allows to have an arbitrary geometry with respect to the underlying global finite elements. Numerical experiments for biomorphic cellular ceramics with porous microstructures produced from natural wood are presented.
\end{abstract}

\section{Global-Local Approach for Heterogeneous Materials}

Consider a domain $\Omega \subset \mathcal{R}^{d}, d=2,3$, occupied by a heterogeneous material with microstructures of periodically distributed constituents. Suppose that the boundary of $\Omega$, denoted by $\Gamma$, consists of a prescribed displacement boundary $\Gamma_{D}\left(\right.$ meas $\left.\Gamma_{D}>0\right)$ and a prescribed traction boundary $\Gamma_{T}$, such that $\Gamma=$ $\Gamma_{D} \cup \Gamma_{T}, \Gamma_{D} \cap \Gamma_{T}=\emptyset$, as shown in Figure 1.

Assume that the periodic cells in the macrostructure are infinitely many but infinitely small and repeated periodically through the medium. The unit microstructure consists of different material constituents and a pore. Both the

\footnotetext{
* This work has been partially supported by the German National Science Foundation (DFG) under Grant No.HO877/5-3. The third author has also been supported in part by the Bulgarian Ministry for Education and Science under Grant I1402/2004.
} 


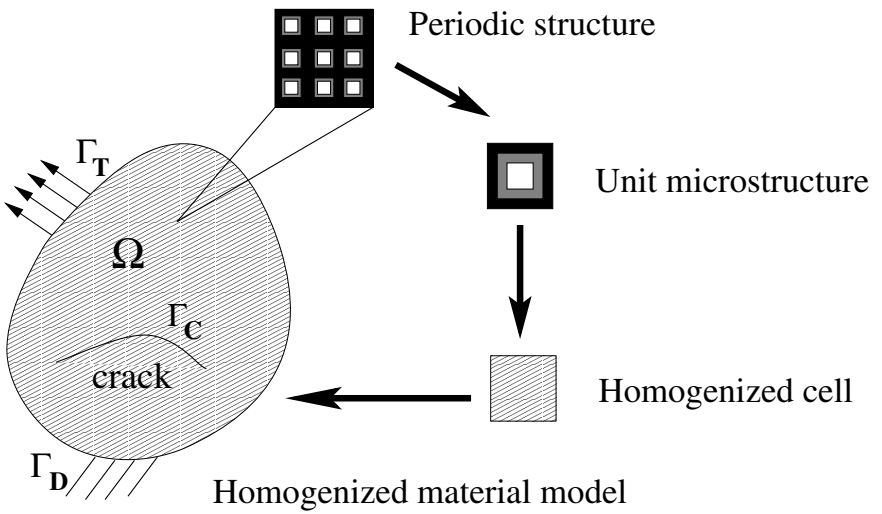

Fig. 1. Crack in the macroscopic homogenized material model

macroscopic and microscopic scales are well separated, i.e., the size of the microstructure in the heterogeneous material is much smaller than those of the macroscopic component. The asymptotic homogenization theory [1]2 is applied to find the effective (homogenized) properties of the material and to derive the homogenized macroscopic model. Details are given in the next Section. The main idea for the homogenization of a heterogeneous material with a periodical distribution of microstructures is illustrated in Figure 1.

We allow the domain $\Omega$ to contain discontinuities and consider the crack problem with a crack $\Gamma_{C}$, see Figure 1. The crack is a multiscale effect which typically appears in regions with microstructures of increasing porosity. The periodicity fails for those microstructures cut by the crack. Therefore, a new finite element analysis has to incorporate microstructural information about the nucleation and growth of micropores. We rely on the mesh superposition method (known as $s$-version of FEM), first developed in 788 . The method is based on a finite element approximation by using two independent meshes: the global mesh for the whole domain (also called in the literature a background mesh) and a local mesh in the critical region near the crack (also called a patch mesh). The local mesh is arbitrarily superimposed onto the global mesh without taking care of the matching between nodes in both meshes.

Consider the following governing equations in the domain $\Omega$

$$
\begin{aligned}
-\nabla \cdot \boldsymbol{\sigma} & =\boldsymbol{b} & & \text { in } \Omega \\
\boldsymbol{u} & =\boldsymbol{g} & & \text { on } \Gamma_{D} \\
\boldsymbol{\sigma} \cdot \boldsymbol{n} & =\boldsymbol{t} & & \text { on } \Gamma_{T},
\end{aligned}
$$

where $\boldsymbol{\sigma}$ is the second order symmetric stress tensor, $\boldsymbol{b}$ is the body force, $\boldsymbol{g}$ is the prescribed displacement on $\Gamma_{D}, \boldsymbol{t}$ is the prescribed traction on $\Gamma_{T}$, and $\boldsymbol{n}$ is the unit normal to the boundary $\Gamma_{T}$. A traction-free surface $\boldsymbol{\sigma} \cdot \boldsymbol{n}=\mathbf{0}$ is assumed on the crack $\Gamma_{C}$. In case of small strains and displacements, the second order strain tensor $\boldsymbol{e}$ is given by 


$$
\boldsymbol{e}=\boldsymbol{e}(\boldsymbol{u})=\left(\nabla \boldsymbol{u}+(\nabla \boldsymbol{u})^{T}\right) / 2,
$$

where $\nabla \boldsymbol{u}$ is the gradient operator. If a linear elasticity is assumed, the constitutive relation is presented by the linearized Hooke law $\boldsymbol{\sigma}=\boldsymbol{E}: \boldsymbol{e}$, where $\boldsymbol{E}$ is the fourth-order elasticity tensor which depends on the material constants like Young's modulus and Poisson's ratio.

The weak form of the governing equation (11) reads: Find $\boldsymbol{u} \in U$, such that

$$
\int_{\Omega} \boldsymbol{e}(\boldsymbol{v}): \boldsymbol{\sigma}(\boldsymbol{u}) d \Omega=\int_{\Omega} \boldsymbol{b} \cdot \boldsymbol{v} d \Omega+\int_{\Gamma_{T}} \boldsymbol{t} \cdot \boldsymbol{v} d \Gamma, \quad \forall \boldsymbol{v} \in U_{0},
$$

where the set of admissible displacement fields is defined by

$$
U=\left\{\boldsymbol{v} \mid \boldsymbol{v} \in V, \boldsymbol{v}=\boldsymbol{g} \text { on } \Gamma_{D}, \boldsymbol{v} \text { discontinuous on } \Gamma_{C}\right\}
$$

and the test function space is defined by

$$
U_{0}=\left\{\boldsymbol{v} \mid \boldsymbol{v} \in V, \boldsymbol{v}=\mathbf{0} \text { on } \Gamma_{D}, \boldsymbol{v} \text { discontinuous on } \Gamma_{C}\right\} .
$$

The space $V$ is related to the regularity of the solution in $\Omega$ and allows for discontinuous functions across the crack. At each point $\boldsymbol{x} \in \Omega$ we consider a finite element discretization of (5) with a basis taken from the test function space $U_{0}$ and nodal shape functions $\boldsymbol{N}(\boldsymbol{x})$ constructed by the Galerkin method.

We denote the local critical region onto which the local mesh is superimposed by $\Omega^{L}, \Omega^{L} \subset \Omega$, a subset of $\Omega$, containing the crack. Let $\Omega^{G}=\Omega \backslash \Omega^{L}$ be the rest of the domain excluding discontinuities. The domains $\Omega$ and $\Omega^{L}$ are discretized independently by separate sets of finite elements $\Omega_{e}^{G}$ and $\Omega_{e}^{L}$, such that $\bigcup \Omega_{e}^{G}=\Omega$ and $\bigcup \Omega_{e}^{L}=\Omega^{L}$. Here, the superscript $G$ relates to the global (underlying) mesh and $L$ to the local (superimposed) mesh. $\Gamma^{G L}$ is the boundary between the two meshes excluding external boundaries, i.e., $\Gamma \cap \Gamma^{G L}=\emptyset$.

Let $\boldsymbol{u}^{G}$ be the global displacement field defined in $\Omega$ and $\boldsymbol{u}^{L}$ be the local displacement field defined in the local region $\Omega^{L}$. Note that the superimposed field $\boldsymbol{u}^{L}$ is in general discontinuous due to a discontinuity across the crack faces. The total displacement field $\boldsymbol{u}$ is constructed by superposition of both displacement fields on the separate meshes and can be written as follows

$$
\boldsymbol{u}= \begin{cases}\boldsymbol{u}^{G} & \text { on } \Omega^{G}, \Gamma^{G L} \\ \boldsymbol{u}^{G}+\boldsymbol{u}^{L} & \text { on } \Omega^{L}\end{cases}
$$

To insure displacement compatibility between the global and local meshes, we assume homogeneous boundary conditions on the boundary of the patch, i.e., $\boldsymbol{u}^{L}=\mathbf{0}$ on $\Gamma^{G L}$. Denote by $\boldsymbol{B}^{G}$ and $\boldsymbol{B}^{L}$ the discretized gradient operators (also called strain-displacement matrices) for the global and local meshes, respectively. Then, the strain can be expressed as follows

$$
e= \begin{cases}e^{G}=\boldsymbol{B}^{G} \boldsymbol{u}^{G} & \text { on } \Omega^{G} \\ e^{L}=\boldsymbol{B}^{G} \boldsymbol{u}^{G}+\boldsymbol{B}^{L} \boldsymbol{u}^{L} & \text { on } \Omega^{L} .\end{cases}
$$


Based on the Hooke law we get the following constitutive relations

$$
\boldsymbol{\sigma}=\boldsymbol{E}: \boldsymbol{e}= \begin{cases}\boldsymbol{\sigma}^{G}=\boldsymbol{E}^{G}:\left(\boldsymbol{B}^{G} \boldsymbol{u}^{G}\right) & \text { on } \Omega^{G} \\ \boldsymbol{\sigma}^{L}=\boldsymbol{E}^{L}:\left(\boldsymbol{B}^{G} \boldsymbol{u}^{G}+\boldsymbol{B}^{L} \boldsymbol{u}^{L}\right) & \text { on } \Omega^{L},\end{cases}
$$

where $\boldsymbol{E}^{G}$ and $\boldsymbol{E}^{L}$ are the elasticity tensors corresponding to the different constitutive laws. By using shape functions $\boldsymbol{N}^{G}(\boldsymbol{x})$ on the global mesh and shape functions $\boldsymbol{N}^{L}(\boldsymbol{x})$ on the local mesh, one can get from the standard weak form (5) the following two equations

$$
\begin{aligned}
\int_{\Omega^{G}} \boldsymbol{B}^{G}(\boldsymbol{x}): \boldsymbol{\sigma}^{G}(\boldsymbol{u}) d \Omega & =\int_{\Omega^{G}} \boldsymbol{N}^{G}(\boldsymbol{x}) \cdot \boldsymbol{b} d \Omega+\int_{\Gamma_{T}^{G}} \boldsymbol{N}^{G}(\boldsymbol{x}) \cdot \boldsymbol{t} d \Gamma, \\
\int_{\Omega^{L}} \boldsymbol{B}^{L}(\boldsymbol{x}): \boldsymbol{\sigma}^{L}(\boldsymbol{u}) d \Omega & =\int_{\Omega^{L}} \boldsymbol{N}^{L}(\boldsymbol{x}) \cdot \boldsymbol{b} d \Omega+\int_{\Gamma_{T}^{L}} \boldsymbol{N}^{L}(\boldsymbol{x}) \cdot \boldsymbol{t} d \Gamma,
\end{aligned}
$$

where $\Gamma_{T}^{G}=\Gamma_{T} \cap \Omega^{G}$ and $\Gamma_{T}^{L}=\Gamma_{T} \cap \Omega^{L}$. Substituting (8)-(10) in equations (11)-(12), we obtain the following discrete system

$$
\left[\begin{array}{ll}
\boldsymbol{K}^{G} & \boldsymbol{K}^{G L} \\
\left(\boldsymbol{K}^{G L}\right)^{T} & \boldsymbol{K}^{L}
\end{array}\right]\left\{\begin{array}{l}
\boldsymbol{u}^{G} \\
\boldsymbol{u}^{L}
\end{array}\right\}=\left\{\begin{array}{l}
\boldsymbol{f}^{G} \\
\boldsymbol{f}^{L}
\end{array}\right\}
$$

where $\boldsymbol{K}^{G}$ and $\boldsymbol{K}^{L}$ are the stiffness matrices corresponding to the global and local meshes, respectively, and $\boldsymbol{K}^{G L}$ is the matrix corresponding to the interaction between the two meshes, namely

$$
\begin{gathered}
\boldsymbol{K}^{G}=\int_{\Omega^{G}}\left(\boldsymbol{B}^{G}(\boldsymbol{x})\right)^{T} \boldsymbol{E}^{G} \boldsymbol{B}^{G}(\boldsymbol{x}) d \Omega+\int_{\Omega^{L}}\left(\boldsymbol{B}^{G}(\boldsymbol{x})\right)^{T} \boldsymbol{E}^{L} \boldsymbol{B}^{G}(\boldsymbol{x}) d \Omega, \\
\boldsymbol{K}^{G L}=\int_{\Omega^{L}}\left(\boldsymbol{B}^{G}(\boldsymbol{x})\right)^{T} \boldsymbol{E}^{L} \boldsymbol{B}^{L}(\boldsymbol{x}) d \Omega, \quad \boldsymbol{K}^{L}=\int_{\Omega^{L}}\left(\boldsymbol{B}^{L}(\boldsymbol{x})\right)^{T} \boldsymbol{E}^{L} \boldsymbol{B}^{L}(\boldsymbol{x}) d \Omega .
\end{gathered}
$$

The force vectors $\boldsymbol{f}^{G}$ and $\boldsymbol{f}^{L}$ are computed from the right-hand sides of (11) and (12), respectively. Note that $\boldsymbol{N}^{G}(\boldsymbol{x})$ are the shape functions corresponding to finite elements in the global mesh on which continuous displacement field $\boldsymbol{u}^{G}$ is considered. Furthermore, $\boldsymbol{N}^{L}(\boldsymbol{x})$ are the discontinuous shape functions of the elements chosen on the local domain to model the crack. The elements of the global and local meshes should not coincide. The cracked mesh is superimposed on the continuous mesh in $\Omega^{L}$ by using the $s$-method. The main difficulty of this method is the numerical integration based on Gauss quadratures when solving the system (13). An attractive approach is recently proposed in [10] where only the near-tip crack fields are modeled on a superimposed patch (overlaid mesh) and the rest of the crack is treated within the framework of the eXtended Finite Element Method (XFEM) by introducing additional discontinuous enrichment functions for the elements completely cut by the crack, see [11]. The latter method is also used in our numerical experiments and briefly explained in Section 3 . 


\section{Multi-Scale Method}

In this section, we describe the multi-scale method based on the asymptotic homogenization theory together with the mesh superposition method. For more details we refer the reader to [16. The homogenization approach is used on the global domain excluding the vicinity of the crack where the periodicity of the microstructures is lost and this approach is not applicable. The crack is considered in the local domain (patch). Two independent (global and local) meshes are generated in the global and local domains, respectively. The patch is allowed to have an arbitrary geometry with respect to the underlying global finite elements. The total displacement field (8) is approximated by adding global (underlying) and local (superimposed) fields and hence, it is discontinuous across the crack.

A double-scale asymptotic expansion for the displacement field and a homogenization procedure by taking a zero limit of the scale ratio are applied to come up with computationally feasible macromodels [1/2. The homogenized macroscopic problem is involved in the governing equation (11). Furthermore, introducing global and local meshes, the global displacement field $\boldsymbol{u}^{G}$ is expressed by the homogenized displacement, the leading term in the asymptotic expansion form (see, e.g., 9]). The homogenized elasticity problem is transformed to solving the system (13) with a symmetric and usually, sparse, stiffness matrix coupling the integrands on the global and local meshes. Note that the elasticity tensor $\boldsymbol{E}^{G}$ in the expression (14) is replaced now by the homogenized elasticity tensor $\boldsymbol{E}^{H}$ with components computed as follows

$$
E_{i j k l}^{H}=\frac{1}{|Y|} \int_{Y}\left(E_{i j k l}(\boldsymbol{y})-E_{i j p q}(\boldsymbol{y}) \frac{\partial \xi_{p}^{k l}}{\partial y_{q}}\right) d Y,
$$

where $E_{i j k l}$ are the elasticity coefficients corresponding to the different material layers in the microstructure. The periodic functions $\boldsymbol{\xi}^{k l}$ (also referred to as characteristic displacements) satisfy the following elasticity equation in the microscopic unit cell

$$
\int_{Y} E_{i j p q}(\boldsymbol{y}) \frac{\partial \xi_{p}^{k l}}{\partial y_{q}} \frac{\partial \phi_{i}}{\partial y_{j}} d Y=\int_{Y} E_{i j k l}(\boldsymbol{y}) \frac{\partial \phi_{i}}{\partial y_{j}} d Y
$$

where $\phi \in \boldsymbol{H}^{1}(Y)$ is an arbitrary $Y$-periodic variational function. The multiscale procedure is realized by the following Multi-Scale Algorithm (MSA)

Step 1. Select a unit microstructure $Y$ in the heterogeneous material.

Step 2. Solve (17) to find the characteristic displacement fields $\boldsymbol{\xi}^{k l}$.

Step 3. Compute the homogenized coefficients (16) and set $\boldsymbol{E}^{G}=\boldsymbol{E}^{H}$.

Step 4. Generate a global mesh in $\Omega$ on the macroscopic homogenized model.

Step 5. Introduce a local (discontinuous) mesh near the crack $\Gamma_{C}$.

Step 6. Solve (13) to determine the displacements $\boldsymbol{u}^{G}$ and $\boldsymbol{u}^{L}$.

Step 7. Substitute $\boldsymbol{u}^{G}$ and $\boldsymbol{u}^{L}$ in (9) and (10) to find the strains and stresses. 


\section{Crack Modeling with Partition of Unity Enrichment}

The extended finite element method 3[11]5] allows treating crack problems without meshing the discontinuity surface. This is possible through enrichment of the standard polynomial finite element space with special functions: discontinuous, to account for the displacement jump, and crack-tip fields to reduce the mesh density required for accurate fracture parameter computations. The method is now becoming quite mature, and has already been applied to industrial fracture mechanics problems [5].

Some elements are split by the crack and others contain the crack tips. Nodes whose support is cut by a crack are in set $\mathcal{N}_{c r}$, while nodes whose support contains one tip are in the set $\mathcal{N}_{\text {tip }}$, as in Figure 2,
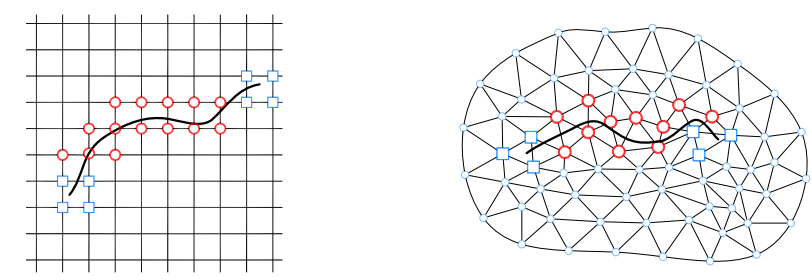

Fig. 2. Selection of enriched nodes. Circled nodes (set of nodes $\mathcal{N}_{c r}$ ) are enriched with the step function whereas the squared nodes (set of nodes $\mathcal{N}_{t i p}$ ) are enriched with the crack tip functions: a) on structured mesh; b) on unstructured mesh.

The XFEM approximation reads

$$
\begin{aligned}
\mathbf{u}^{h}(\mathbf{x}) & =\sum_{I \in \mathcal{N}} N_{I}(\mathbf{x}) \mathbf{u}_{I}+\sum_{J \in \mathcal{N}_{c r}} \tilde{N}_{J}(\mathbf{x})\left(H(\mathbf{x})-H\left(\mathbf{x}_{J}\right)\right) \mathbf{a}_{J} \\
& +\sum_{K \in \mathcal{N}_{t i p}} \tilde{N}_{K}(\mathbf{x}) \sum_{\alpha=1}^{4}\left(B_{\alpha}(\mathbf{x})-B_{\alpha}\left(\mathbf{x}_{K}\right)\right) \mathbf{b}_{\alpha K}
\end{aligned}
$$

where $N_{I}(\mathbf{x})$ and $\tilde{N}_{J}(\mathbf{x})$ are finite element shape functions, while $\mathbf{u}_{\mathrm{I}}, \mathbf{a}_{\mathrm{J}}$ and $\mathbf{b}_{\alpha \mathrm{K}}$ are the displacement and enrichment nodal variables, respectively. Note that the shape functions $\widetilde{N}_{J}(\mathbf{x})$ associated with the enrichment can differ from the shape functions $N_{I}(\mathbf{x})$ used for the standard part of the displacement approximation. $H(\mathbf{x})$ is the modified Heaviside function which takes on the value +1 above the crack and -1 below the crack and $B_{\alpha}(\mathbf{x})$ is a basis that spans the near tip asymptotic field:

$$
\mathbf{B} \equiv\left[B_{1}, B_{2}, B_{3}, B_{4}\right]=\left[\sqrt{r} \sin \frac{\theta}{2}, \sqrt{r} \cos \frac{\theta}{2}, \sqrt{r} \sin \frac{\theta}{2} \cos \theta, \sqrt{r} \cos \frac{\theta}{2} \cos \theta\right]
$$

From the enriched approximation (18), Bubnov-Galerkin procedure gives discrete equations of the form $\mathbf{K d}=\mathbf{f}$. Numerical integration for split elements is done here by partitioning the elements into sub-triangles. Interested readers can refer to [413] for details. 


\section{Numerical Experiments}

The fracture mechanics computations in this section are performed using the Xfem library OpenXfem $++[6]^{1}$. Our numerical examples concern biomorphic silicon carbide $(\mathrm{SiC})$ cellular ceramics with porous microstructures produced from natural wood. The open porous system of tracheidal cells which provide the transportation path for water and minerals in the living plants is accessible for infiltration of various liquid or gaseous metals (see, e.g., [14 for the production process). Numerical experiments for the homogenized coefficients in a 2-D material workpiece are given in 9 .

Consider a stationary microstructure with a geometrically simple tracheidal periodicity cell $Y=[0,1]^{2}$ consisting of an outer layer of carbon (C), interior layer of silicon carbide $(\mathrm{SiC})$, and a centered pore channel ( $\mathrm{P}$, no material), see Figure 3h). One can also deal with the so-called pure $\mathrm{SiC}$ ceramics when enough silicon is infiltrated in the pore channel until the complete reaction between the carbon and silicon, see Figure 3b). The Young modulus $E$ (in GPa) and the Poisson ratio $\nu$ of our two materials are, respectively, $E=10, \nu=0.22$ for carbon and $E=410, \nu=0.14$ for SiC.
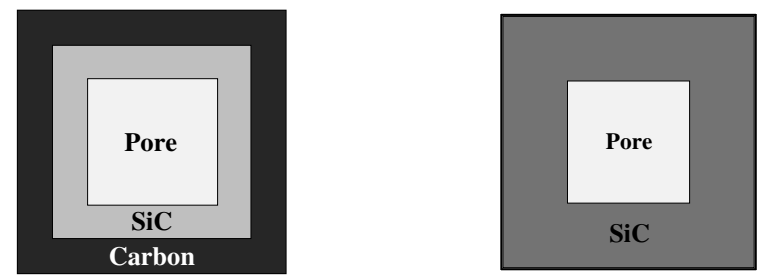

Fig. 3. a) Unit cell $Y=P \cup S i C \cup C$; b) Pure SiC ceramics: $Y=P \cup S i C$

As a first attempt we consider a crack in the macroscopic homogenized model. All computations are performed assuming the theory of Linear Elastic Fracture Mechanics. In Table 1 we report the evaluated data for the homogenized Young modulus $E^{H}$ (in GPa) and the homogenized Poisson ratio $\nu^{H}$. In case of $\mathrm{SiC}$ ceramics the coefficients are computed for equal widths of the $\mathrm{SiC}$ and carbon layers, see Table 1 $\mathrm{a}$ ). The density of the $\mathrm{SiC}$ layer and the density of the carbon layer are denoted, respectively, by $\mu_{S i C}$ and $\mu_{\text {carbon }}$. Note that the porosity is determined by: $\operatorname{meas}(Y)-\mu_{\text {SiC }}-\mu_{\text {carbon }}$. In case of pure $\mathrm{SiC}$ ceramics, see Table 1b), the porosity is given by: $\operatorname{meas}(Y)-\mu_{S i C}$.

In all problems, the energy release rate $G=\frac{1}{E^{\prime}}\left(K_{I}^{2}+K_{I I}^{2}\right)$ with $E^{\prime}=$ $E^{H} /\left(1-\left(\nu^{H}\right)^{2}\right)$ is computed for both crack tips. The stress intensity factors (SIFs), $K_{I}$ and $K_{I I}$, for mode I and II, respectively, are determined using the domain form of the interaction integrals [12. We are interested in the effect of porosity on the energy release rate. Consider a center crack in an infinite plate under remote, unit, uniaxial tension. The geometry of the plate is $(2 \times 6)$, the

\footnotetext{
${ }^{1}$ Available on request.
} 
Table 1. Homogenized $E$ and $\nu$ : a) SiC ceramics; b) Pure SiC ceramics

\begin{tabular}{|l|l|l|l|l|}
\hline porosity & $\mu_{\text {SiC }}$ & $\mu_{\text {carbon }}$ & $E^{H}(\mathrm{GPa})$ & $\nu^{H}$ \\
\hline 0.81 & 0.0925 & 0.0925 & 89.42 & 0.035 \\
0.64 & 0.17 & 0.19 & 66.52 & 0.069 \\
0.36 & 0.28 & 0.36 & 43.47 & 0.142 \\
0.25 & 0.3125 & 0.4375 & 37.29 & 0.145 \\
0.16 & 0.33 & 0.51 & 31.31 & 0.137 \\
0.09 & 0.3325 & 0.5775 & 25.99 & 0.140 \\
0.04 & 0.32 & 0.64 & 21.69 & 0.153 \\
0.01 & 0.2925 & 0.6975 & 18.56 & 0.168 \\
\hline
\end{tabular}

\begin{tabular}{l|l|l|l|}
\hline porosity & $\mu_{S i C}$ & $E^{H}(\mathrm{GPa})$ & $\nu^{H}$ \\
\hline 0.9025 & 0.0975 & 216.10 & 0.016 \\
0.7225 & 0.2775 & 231.94 & 0.040 \\
0.5625 & 0.4375 & 248.93 & 0.062 \\
0.4225 & 0.5775 & 267.94 & 0.083 \\
0.3025 & 0.6975 & 289.27 & 0.104 \\
0.2025 & 0.7975 & 314.16 & 0.124 \\
0.1225 & 0.8775 & 342.27 & 0.138 \\
0.0025 & 0.9975 & 409.14 & 0.139 \\
\hline
\end{tabular}

crack length is 0.25 , and the mesh, completely regular and non-conforming to the crack, contains 2701 four-noded quadrilateral elements. The radius of the circular interaction integral domain is twice the size of the element containing the tip. Note that in this case, the exact SIFs are actually known analytically. The computed SIFs are within one percent of the exact values. The evolution of the energy release rate $G$ as a function of porosity is given in Figure $4 \mathrm{~b}$ ) and shows that, as expected, the energy release rate increases with increasing porosity, i.e. as the pure $\mathrm{SiC}$ ceramics become more rigid.
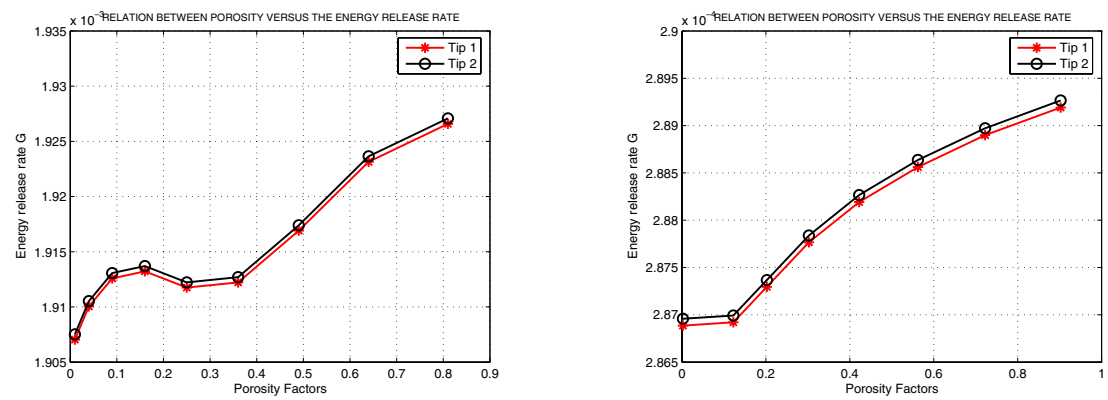

Fig. 4. Porosity versus the energy release rate $G$ : a) SiC ceramics; b) Pure SiC ceramics

The same problem was solved for a layered $\mathrm{SiC}$ ceramics, and results are reported in Figure 4a). We note that the behavior is somewhat more complex in this case, where we observe a decreasing energy release rate for porosities comprised between 0.15 and 0.25 .

\section{References}

1. Bensoussan, A., Lions, J.L., Papanicolaou, G.: Asymptotic Analysis for Periodic Structures. North-Holland, Elsevier Science Publishers, Amsterdam, (1978).

2. Jikov, V.V., Kozlov, S.M., Oleinik, O.A.: Homogenization of Differential Operators and Integral Functionals. Springer, (1994). 
3. Belytschko T., Moës N., Usui S., Parimi C.: Arbitrary discontinuities in finite elements. Int. J. Numer. Methods Eng., 50 (4) (2001) 993-1013.

4. Bordas S., Legay A.: Enriched finite element short course: class notes. Organized by S. Bordas and A. Legay through the EPFL school of continuing education, Lausanne, Switzerland, December 7-9, 2005.

5. Bordas S., Moran B.: Extended finite element and level set method for damage tolerance assessment of complex structures. Eng. Fract. Mech., 73 (9) (2006) 11761201.

6. Bordas S., Nguyen V.P., Dunant C., Nguyen-Dang H., Guidoum A.: An objectoriented extended finite element library. Int. J. Numer. Methods Eng., (2006) (to appear).

7. Fish J.: The $s$-version of the finite element method. Comput. Struct., 43 (3) (1992) 539-547.

8. Fish J., Guttal R.: The $s$-version of finite element method for laminated composites. Int. J. Numer. Methods Eng., 39 (21) (1996) 3641-3662.

9. Hoppe R.H.W., Petrova S.I.: Optimal shape design in biomimetics based on homogenization and adaptivity. Math. Comput. Simul., 65 (3) (2004) 257-272.

10. Lee S.-H., Song J.-H., Yoon Y.-C., Zi G., Belytschko T.: Combined extended and superimposed finite element method for cracks. Int. J. Numer. Methods Eng., 59 (8) (2004) 1119-1136

11. Moës N., Dolbow J., Belytschko T.: A finite element method for crack growth without remeshing. Int. J. Numer. Methods Eng., 46 (1) (1999) 131-150.

12. Moran, B., Shih, C.F.: Crack tip and associated domain integrals from momentum and energy balance. Eng. Fract. Mech., 27 (1987) 615-641.

13. Nguyen V.P.: An object oriented approach to the X-FEM with applications to fracture mechanics. Master's thesis, EMMC-Hochiminh University of Technology, Vietnam, November (2005).

14. Ota T., Takahashi M., Hibi T., Ozawa M., Suzuki S., Hikichi Y., Suzuki H.: Biomimetic process for producing SiC wood. J. Amer. Ceram. Soc., 78 (1995) 3409-3411.

15. Sukumar N., Prévost J.-H.: Modeling quasi-static crack growth with the extended finite element method. Part I: Computer implementation. Int. J. Solids Struct., 40 (2003) 7513-7537.

16. Takano N., Zako M., Okuno Y.: Multi-scale finite element analysis of porous materials and components by asymptotic homogenization theory and enhanced mesh superposition method. Modelling Simul. Mater. Sci. Eng., 11 (2003) 137-156. 